\title{
Comparison of long-term variations of the cosmic ray flux from the network of ground-based detectors, PAMELA and AMS-02 data
}

\author{
Anatoly V. Belov ${ }^{\circledR}$, Victor G. Yanke ${ }^{\circledR}$, Raisa Gushchina ${ }^{\circledR}$, Nataly Shlyk \\ Correspondence \\ Pushkov Institute of Terrestrial Magnetism, lonosphere and Radio Wave Propagation, (IZMIRAN) Moscow, Russia, \\ abelov@izmiran.ru, yanke@izmiran.ru,rgus@izmiran.ru,nshlyk@izmiran.ru
}

\section{OPEN ACCESS}

This work is published under the Creative Commons Attribution 4.0 International license (CC BY 4.0)

Please note that individual, appropriately marked parts of the work may be excluded from the licens mentioned or may be subject to other copyright conditions. If such third party material is not under the Creative Commons license, any copying, editing or public reproduction is only permitted with the prior consent of the respective copyright owner or on the basis of relevant legal authorization regulations.

\section{Keywords}

long-term variations; PAMELA, AMS-02; ground-based detectors; balloon stratospheric sounding

\begin{abstract}
The paper presents preliminary results of a comparison of long-term variations of the cosmic ray flux using data from the network of ground-based detectors with direct flux measurements on the PAMELA and AMS-02 magnetic spectrometers and a series of balloon stratospheric soundings. The analysis showed good agreement for the entire period of continuous ground-based monitoring of cosmic ray variations.
\end{abstract}

\section{Introduction}

Galactic cosmic rays with rigidity up to one hundred GV are constantly modulated by the solar wind. On the one hand, this galactic cosmic rays flux is recorded by a network of ground-based detectors under the atmosphere: ionization chambers, muon telescopes, and neutron monitors. Cosmic ray variations are determined experimentally from ground-based observations at the network of cosmic ray stations, and the processing task is reduced to restoring variations in the near-Earth interplanetary space from the observational data. This problem is solved by the global spectrographical method (GSM) (Krymsky et al. 1966; Nagashima 1971; Belov et al. 1983).

Recently, more and more often, the flux of galactic cosmic rays is measured directly by spacecraft detectors at various points of the heliosphere and near the Earth's orbit.

The aim of this work is to compare the results of ground-based measurements processed by the GSM method with the direct measurements of the cosmic ray flux on spacecrafts. Such an opportunity appeared only with the launch of the PAMELA and AMS-02 magnetic spectrometers, which measure particle fluxes in a wide range of rigidities, including the effective particle rigidity of the ground network of detectors - $10 \mathrm{GV}$.

There are quite a lot of works with comparisons of direct and indirect measurements (Alanko et al. 2003; Usoskin et al. 2005, 2011, 2017; Koldobskiy et al. 2018, 2019a, 2019b). True, the ultimate goal of these works was to reconstruct the modulation potential within the force field approximation. Thus, in Usoskin et. al. 2005, the era of ground-based monitoring of cosmic radiation from 1951 to 2004 was considered. The reconstructed spectrum of cosmic rays from several neutron monitors was calibrated using the data of balloon stratospheric sounding and AMS-01. The calibrated spectrum was used to reconstruct the modulation potential values. In Usoskin et al. 2017, the data series was extended to 2010 and the data from the PAMELA magnetometer were also used for calibration. 
In Usoskin et al. 2011, several epochs of ground-based monitoring of cosmic radiation were considered. The period of ionization chambers (Forbush) from 1936 to 1951, the period of single neutron monitors IGY (mining) from 1951 to 1964 and the modern period of operation of the network of neutron monitors nm64, although the above-mentioned works used data from only 6-8 neutron detectors. For the same period, the modulation potential was also reconstructed from the data of stratospheric sounding. It is hoped that the use of data from detectors of the entire world network ( $>40$ ) should increase the accuracy, expand the energy range, and remove some uncertainties in the obtained results.

\section{Ground monitoring and global spectrographic method GSM}

The Earth is a giant natural magnetic spectrometer that separates primary charged particles according to their rigidity, so that registration of cosmic rays at different latitudes and depths in the atmosphere gives significantly different results. The ground-based network of detectors consists of more than 40 neutron monitors, 3 stratospheric sounding stations, and a dozen multi-directional muon telescopes.

The counting rates of the detectors $N$ are directly measurable. The measured variations in the zero-harmonic approximation are related to the spectrum of primary variations reflecting interplanetary processes $\delta J / J_{B}(R)$ by the system of Fredholm integral equations of the first kind:

$$
v^{i}=\delta N /\left.N\right|_{R_{c}^{i}}=\int W\left(R_{c}^{i}, h_{0}^{i}, R\right) \cdot \delta J / J_{B} \cdot \mathrm{dR},
$$

where $\mathrm{i}=1, \ldots, \mathrm{m}$ defines the number of the detector. Here, the coupling function $W^{i}\left(R_{c}^{i}, h_{0}^{i}, R\right)$ between primary and secondary variations recorded by detector $i$, located at point with the rigidity of geomagnetic cutoff $R_{c}^{i}$ at a depth in the atmosphere $h_{0}^{i}$ acts as the kernel of the equation, and the spectrum of variations $d J / J(R)$ - as an unknown function. In our case, the circumstance is that the desired solution can be sought in the form of an analytical function of the spectrum of variations $d J / J$ with a certain number of parameters, which increases the stability of the found solution.

For the spectrum of variations, a parametric representation is often used in the form

$$
d J / J_{B}=a_{1}\left(R_{0}+R\right)^{-\gamma} \text { at } R \leq R_{u},
$$

in (Belov et al. 1998) a variant of a global spectrographical method based on monthly average data is described, specially adapted for studying long-term variations in the approximation of isotropic variations.

As a result of the calculations performed for the period of cosmic ray monitoring, the amplitude and parameters of the spectrum of variations of the zero harmonic were obtained for a rigidity of $10 \mathrm{GV}$, which is close to the effective rigidity of particles recorded by neutron monitors. The result of this analysis can be found in (Yanke et al. 2020).

\section{Data from the PAMELA and AMS-02 magnetic spectrometers}

Direct measurements of galactic cosmic rays, including considered range of rigidities near $10 \mathrm{GV}$, were carried out using orbital detectors on the PAMELA and AMS-02 spacecraft.

The PAMELA (Adrianiet et al. 2011, 2013, 2014, 2017) (elliptical orbit 350-600 km) operated from summer 2006 to January 2014. The geometric factor of the PAMELA magnetic spectrometer is $21.5 \mathrm{~cm}^{2} \mathrm{sr}$ (Adrianiet et al. 2017). The PAMELA operating period is divided into 83 time slots. Each time slot contains 78 energy bands.

The AMS-02 (Aguilar et al. 2015, 2018) is a magnetic spectrometer installed on board of the ISS and has been operating from 2011 to the present. Its operating period is divided into 79 time intervals. 
Each time slot contains 45 energy bands. The geometric factor of the AMS-02 spectrometer is $\sim 0.5$ $\mathrm{m}^{2}$ sr (Ting 2013).

The digital data of the PAMELA and AMS-02 detectors, averaged over the Carrington rotations, are available in the database (crdb 2020) and are described in (Di Felice et al. 2017). Sampling by the time or by the rigidity $(\mathrm{t}, \mathrm{R})$ is possible.

In this work energy channels close to $10 \mathrm{GV}$ are important for comparison with the results of the World Network of Stations (CR Network 2020). The considered energy ranges of the PAMELA and AMS-02 detectors are given in Table 1, which also shows the average energy/rigidity values for each range.

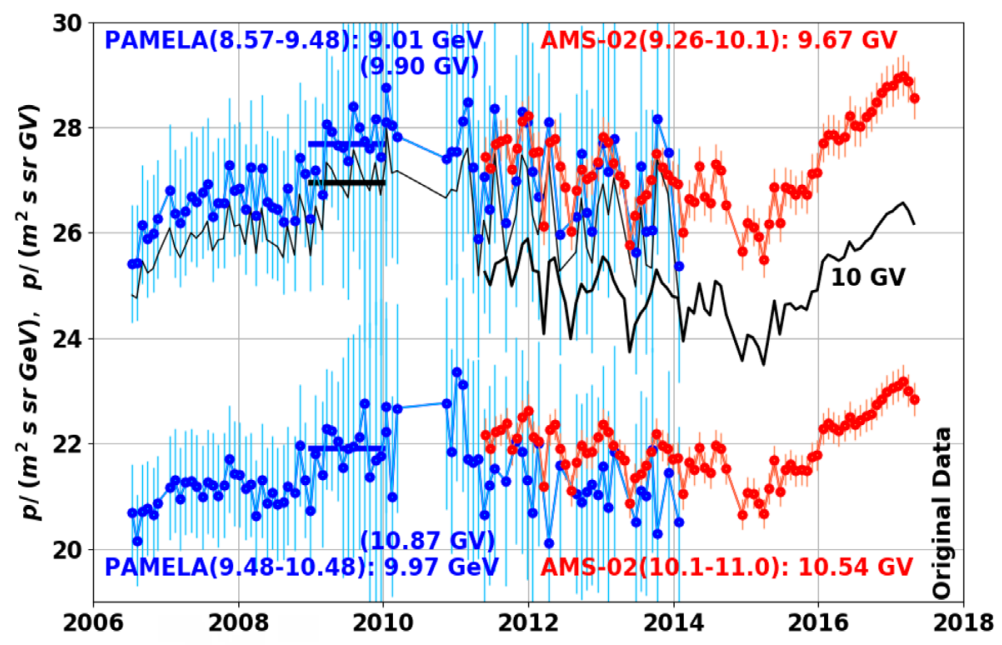

Figure 1: - a10 (\% to 2009) - variations of the density of CRs with a rigidity of $10 \mathrm{GV}$ (red curve with dots in the lower).

Figure 1 shows the time course of the energy ranges of the PAMELA and AMS-02 magnetic spectrometers selected in accordance with the table 1 . The statistical errors of the PAMELA spectrometer data up to 2009 for the considered energies of $\sim 10 \mathrm{GeV}$ are about $4 \%$. Subsequently, the statistical error of the PAMELA data in this range almost doubled. With the same methodology, this indicates a hardware problem. The statistical errors of the data of the AMS-02 spectrometer are significantly lower and slightly exceed $1 \%$ for the rigidity of $~ 10 \mathrm{GV}$ for the entire monitoring period. The solar activity minimum in 2009 was chosen as the base period. It is shown in figure 1 by horizontal lines. The thin black curve shows temporal changes in particle flux recalculated for $10 \mathrm{GV}$ for the PAMELA. The double black curve shows the AMS-02 data recalculated for $10 \mathrm{GV}$. The time dependence of the proton flux reduced to $10 \mathrm{GV}$ is also shown. Temporal changes for the $10 \mathrm{GV}$ rigidity will be discussed below.

\begin{tabular}{|l|l|l|l|}
\hline \multicolumn{2}{|l|}{ PAMELA } & AMS-02 \\
\hline range, GeV & $\mathrm{E}_{\text {evr' }}$ GeV/R $\mathrm{R}_{\text {evr }} \mathrm{GV}$ & range, $\mathrm{GV}$ & $\mathrm{R}_{\text {evr' }} \mathrm{GV}$ \\
\hline $8.57 \div 9.48$ & $9.01 / 9.90$ & $9.26 \div 10.1$ & $\mathbf{9 . 6 7}$ \\
\hline $9.48 \div 10.48$ & $9.97 / 10.87$ & $10.1 \div 11.0$ & $\mathbf{1 0 . 5 4}$ \\
\hline
\end{tabular}

Table 1: Considered energy ranges of the PAMELA and AMS-02 detectors. 


\section{The analysis method}

In order to compare the long-term changes in the cosmic ray flux from the data of ground-based detectors and the PAMELA and AMS-02 spectrometers, it is necessary to generate the PAMELA and AMS-02 data for the rigidity of $10 \mathrm{GV}$, for which there are reliable ground-based measurements of cosmic rays, and to calibrate the measurements of the unified ground-based cosmic ray detector according to direct measurements.
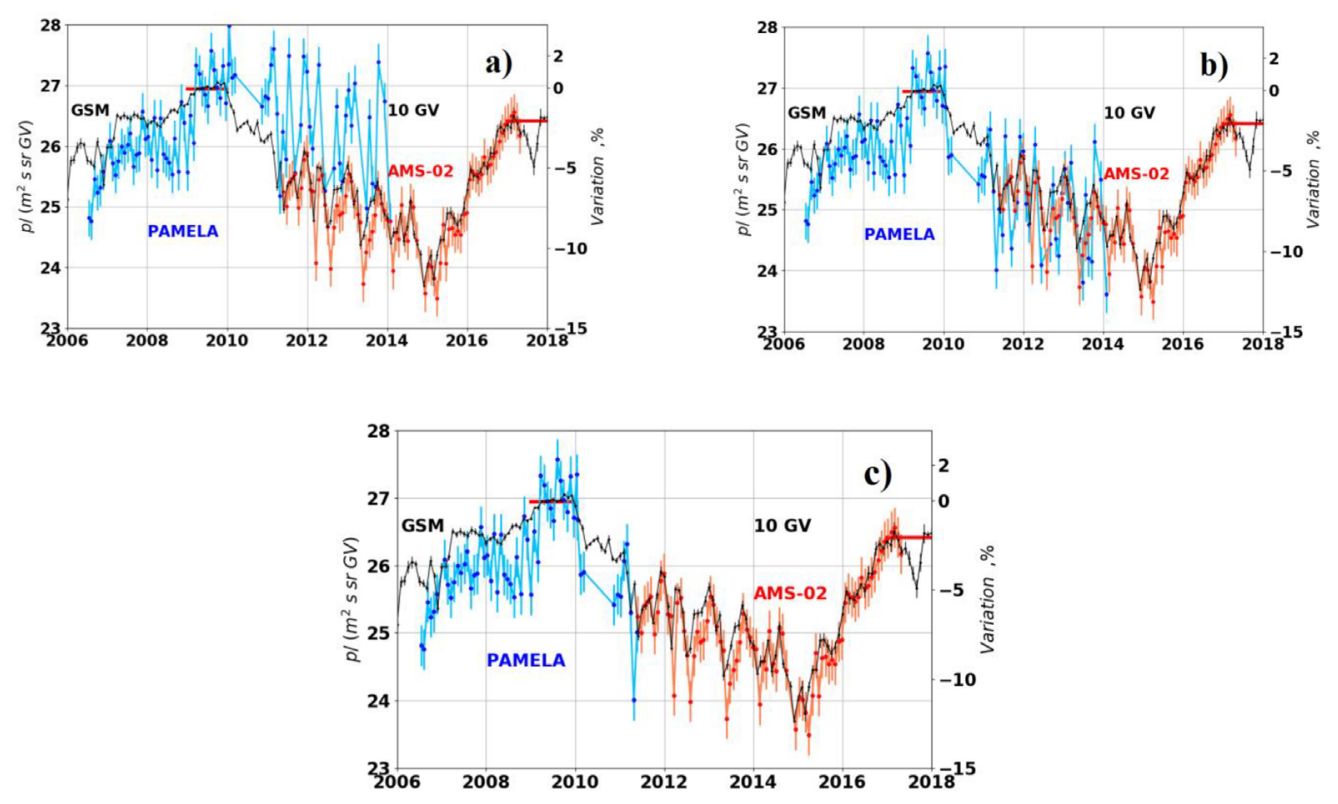

Figure 2: Comparison of the PAMELA and AMS-02 spectrometers data with the GSM data.

The flux of protons is experimentally determined in a certain range of rigidities; therefore, it is necessary to determine the average rigidity of particles in this range. In a limited range of rigidities, spectra can always be represented in a power-law form. By definition, the average rigidity $\bar{R}$ in the case of a power-law spectrum $J=\mathrm{aR}^{-\gamma}$ in the interval $\left[\mathrm{R}_{1}, \mathrm{R}_{2}\right]$ is determined from the equality (Lafferty et al. 1995)

$$
a \cdot \bar{R}^{-\gamma} \cdot\left(R_{2}-R_{1}\right)=a \int R^{-\gamma} \mathrm{dR}
$$

But for calculating the average values $\bar{R}$, the spectrum index $\gamma$ is unknown, which can be determined by solving the system of transcendental equations for two adjacent intervals $\left[R_{1}, R_{2}\right]$ and $\left[\mathrm{R}_{3}, \mathrm{R}_{4}\right]$ and the definition of $\bar{R}$ and $\gamma$.

Since the results of the GSM analysis are cosmic ray variations, it is necessary to directly calibrate and link the data of such a multidirectional ground-based detector to the real spectra of galactic cosmic rays.

The calibration procedure is as follows. Variations relative to the base period with the flow $J_{B}$ during this period are, by definition, equal to

$$
v=\left(J-J_{B}\right) / J_{B}
$$


Variations are determined as a result of the GSM analysis. Then for calibration we get

$$
J=J_{B}(v+1)
$$

The calibration was carried out relative to the base period of 2009 according to the PAMELA magnetometer data, for which $J_{B}=26.94 \mathrm{p} /\left(\mathrm{m}^{2} \mathrm{~s}\right.$ sr GV) for the rigidity of $10 \mathrm{GV}$. The result of this calibration for the entire period of continuous ground-based monitoring of cosmic rays is given in table 2. Statistical errors of the proton flux for the period after 1970 are $\pm 1.2 \mathrm{p} /\left(\mathrm{m}^{2} \mathrm{~s}\right.$ sr GV).

\section{Comparison of the PAMELA and AMS-02 spectrometers data with the GSM data}

In figure $2 \mathrm{a}$ we compared the initial data of the PAMELA and AMS-02 magnetic spectrometers (see figure 1), converted to $10 \mathrm{GV}$, with the calibrated GSM data in flux units (left scale) and their variations (right scale). Variations are calculated relative to the base period 2009 of the PAMELA data. We should note the weak agreement between the PAMELA and GSM data until 2009 and a completely different time course of the PAMELA data from 2010 with very large variations, and a general shift relative to the ground data. The agreement between the AMS-02 and the GSM data is good for the entire presented period. Variations in the data of the AMS-02 magnetometer were also calculated relative to the base period of 2009 using the baseline PAMELA value. Therefore, the agreement of the variations indicates the correct calibration and the absence of drift of the data of the detectors of both magnetic spectrometers at that time.

In figure $2 \mathrm{~b}$, it is assumed that the efficiency of the PAMELA detector after 2010-01-13 is $\varepsilon=$ 1.0488 for $10 \mathrm{GV}$. This problem was also discussed by the authors of the PAMELA project (Martucci et al. 2018). And finally, figure $2 \mathrm{c}$ shows the combined data of the two detectors PAMELA and AMS-02, which generally agree well with the GSM data.

Figure 3 shows the temporal changes of the galactic cosmic ray protons flux at $1 \mathrm{AU}$ according to the data of the ground-based network of neutron monitors and comparison with direct measurements using the PAMELA and AMS-02 spectrometers. Dots also show some data from balloon stratospheric sounding.

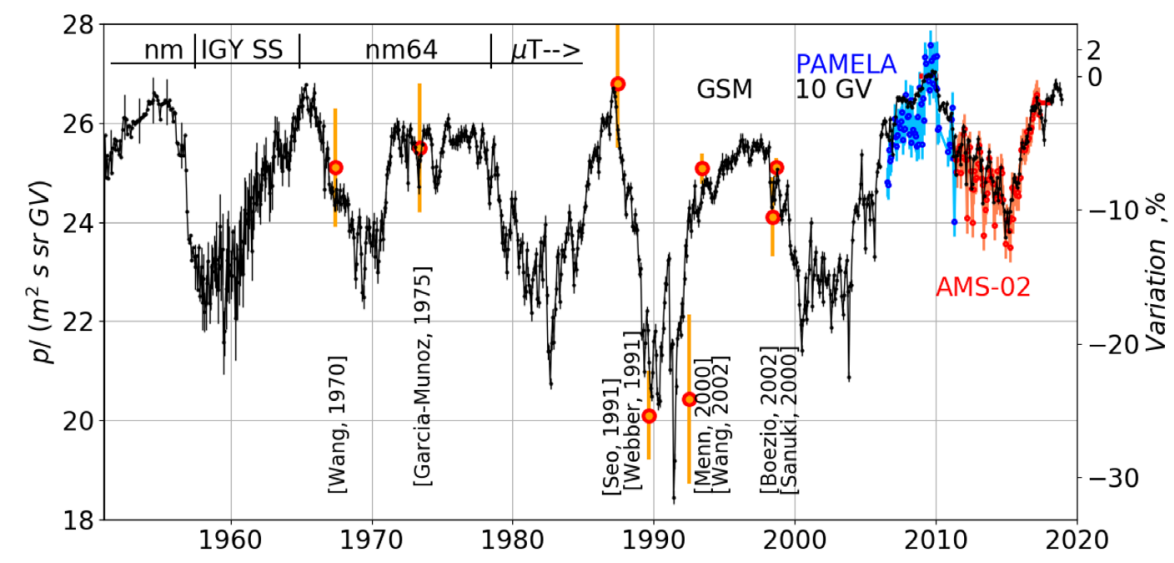

Figure 3: Time variations of the GCR proton flux at 1AU based on the data from the ground-based network of detectors 


\section{Discussion and conclusions}

Due to its unique capabilities, the PAMELA satellite experiment made it possible to calibrate the spectrum of variations obtained as a result of continuous ground monitoring of cosmic ray variations and their GSM processing. Direct measurements of the particle flux in the PAMELA and AMS-02 experiments, as well as the data from a series of balloon stratospheric soundings, made it possible to compare it with the long-term variations of the cosmic ray flux from the network of ground-based detectors data.

The results of continuous ground-based monitoring of cosmic ray variations and the results of direct measurements of the particle flux for the entire observation period are in good agreement. Despite the doubling of the PAMELA measurement error for the period after 2009, no overall drift was observed.

The AMS-02 data for the period 2012-2014 demonstrate large, in comparison with the result of the GSM, short-period variations, which may indicate an underestimation of low energies in the GSM method.

The assessment of the cosmic ray flux outside the magnetosphere for the entire observational period obtained by the GSM method was carried out. Space radiation is a significant obstacle to manned flights. Accurate measurements of cosmic radiation are essential for planning appropriate protection measures. On the basis of ground-based monitoring, fluxes of galactic protons were retrospectively obtained with an average monthly resolution for the period of the space era (see table 2). The calibration was carried out according to the data of the PAMELA magnetic spectrometer in the base period of 2009 The same results can be obtained for the daily average and hourly average resolution.

\begin{tabular}{|c|c|c|c|c|c|c|c|c|c|c|c|c|c|}
\hline year & Jan & Feb & Mar & Apr & May & Jun & Jul & Aug & Sep & Oct & Nov & Dec & Annual \\
\hline 1951 & & 25.76 & 24.90 & 25.35 & 24.89 & 25.27 & 25.41 & 25.00 & 25.49 & 25.67 & 25.46 & 25.65 & 25.35 \\
\hline 1952 & 25.46 & 25.37 & 25.65 & 25.31 & 25.88 & 26.11 & 26.11 & 26.08 & 25.78 & 25.95 & 25.95 & 25.72 & 25.78 \\
\hline 1953 & 25.91 & 26.03 & 25.98 & 26.01 & 26.02 & 26.06 & 26.05 & 26.09 & 26.11 & 26.13 & 26.17 & 26.17 & 26.06 \\
\hline 1954 & 26.17 & 26.28 & 26.45 & 26.39 & 26.43 & 26.41 & 26.46 & 26.61 & 26.56 & 26.54 & 26.46 & 26.38 & 26.43 \\
\hline 1955 & 26.07 & 26.37 & 26.39 & 26.40 & 26.46 & 26.42 & 26.43 & 26.35 & 26.46 & 26.27 & 26.30 & 26.03 & 26.33 \\
\hline 1956 & 26.22 & 26.21 & 25.60 & 25.59 & 25.37 & 25.35 & 25.55 & 25.53 & 25.35 & 25.76 & 25.05 & 24.22 & 25.48 \\
\hline 1957 & 23.65 & 23.58 & 23.73 & 23.23 & 23.49 & 23.31 & 23.10 & 23.33 & 22.64 & 23.49 & 22.51 & 23.10 & 23.03 \\
\hline 1958 & 22.38 & 22.86 & 22.19 & 22.38 & 22.77 & 23.21 & 22.35 & 22.70 & 23.10 & 23.01 & 23.27 & 23.15 & 22.78 \\
\hline 1959 & 23.26 & 22.63 & 23.06 & 23.92 & 23.12 & 23.45 & 21.57 & 21.80 & 22.65 & 23.23 & 23.41 & 23.14 & 22.94 \\
\hline 1960 & 22.94 & 22.91 & 23.81 & 23.28 & 22.06 & 22.81 & 22.79 & 23.19 & 23.46 & 23.63 & 22.96 & 23.59 & 23.12 \\
\hline 1961 & 24.25 & 24.18 & 23.98 & 24.04 & 24.43 & 24.50 & 23.41 & 24.21 & 24.39 & 24.82 & 24.97 & 24.61 & 24.32 \\
\hline 1962 & 24.54 & 24.36 & 24.75 & 24.96 & 25.20 & 24.88 & 25.21 & 25.13 & 25.07 & 24.58 & 24.70 & 24.74 & 24.84 \\
\hline 1963 & 25.31 & 25.32 & 25.83 & 25.80 & 25.48 & 25.67 & 25.37 & 25.33 & 25.04 & 25.31 & 25.57 & 25.33 & 25.45 \\
\hline 1964 & 25.40 & 25.07 & 25.34 & 25.46 & 25.74 & 26.11 & 25.65 & 25.89 & 26.07 & 26.13 & 26.09 & 26.33 & 25.77 \\
\hline 1965 & 26.47 & 26.35 & 26.52 & 26.68 & 26.77 & 26.51 & 26.27 & 26.25 & 26.17 & 26.33 & 26.48 & 26.60 & 26.45 \\
\hline 1966 & 26.12 & 26.12 & 25.96 & 25.86 & 26.01 & 25.64 & 25.62 & 25.93 & 24.66 & 25.26 & 25.48 & 25.29 & 25.66 \\
\hline 1967 & 24.78 & 24.60 & 24.69 & 24.62 & 24.35 & 24.40 & 24.67 & 24.43 & 24.40 & 24.64 & 24.37 & 24.53 & 24.54 \\
\hline 1968 & 24.53 & 24.32 & 24.36 & 24.44 & 24.36 & 23.83 & 23.94 & 24.05 & 23.85 & 23.69 & 22.86 & 23.16 & 23.95 \\
\hline 1969 & 23.76 & 24.10 & 23.89 & 23.35 & 22.58 & 22.49 & 23.07 & 23.59 & 23.90 & 23.85 & 23.89 & 23.58 & 23.50 \\
\hline 1970 & 23.52 & 23.74 & 23.99 & 23.30 & 23.84 & 22.99 & 23.21 & 23.65 & 24.04 & 23.97 & 23.66 & 24.28 & 23.68 \\
\hline 1971 & 24.40 & 24.95 & 25.06 & 25.19 & 25.34 & 25.73 & 25.73 & 25.81 & 25.83 & 25.93 & 25.86 & 25.86 & 25.47 \\
\hline 1972 & 25.50 & 25.35 & 25.72 & 25.91 & 25.66 & 25.24 & 25.76 & 24.79 & 25.84 & 25.88 & 25.60 & 25.59 & 25.57 \\
\hline 1973 & 25.61 & 25.51 & 25.41 & 25.17 & 24.71 & 25.25 & 25.35 & 25.57 & 25.85 & 25.79 & 25.77 & 25.87 & 25.49 \\
\hline 1974 & 25.78 & 25.89 & 25.67 & 25.57 & 25.11 & 24.83 & 24.69 & 25.13 & 24.76 & 24.92 & 25.03 & 25.41 & 25.23 \\
\hline 1975 & 25.43 & 25.69 & 25.74 & 25.95 & 25.97 & 26.08 & 25.91 & 25.64 & 25.59 & 25.66 & 25.58 & 25.60 & 25.74 \\
\hline 1976 & 25.67 & 25.58 & 25.74 & 25.64 & 25.81 & 25.74 & 25.78 & 25.75 & 25.86 & 25.73 & 25.63 & 25.52 & 25.70 \\
\hline 1977 & 25.72 & 25.83 & 25.88 & 25.85 & 25.80 & 25.53 & 25.20 & 25.32 & 25.31 & 25.62 & 25.87 & 25.79 & 25.64 \\
\hline 1978 & 25.42 & 25.27 & 25.46 & 24.89 & 24.39 & 24.71 & 24.91 & 25.52 & 25.56 & 24.97 & 25.10 & 24.97 & 25.10 \\
\hline
\end{tabular}




\begin{tabular}{|c|c|c|c|c|c|c|c|c|c|c|c|c|c|}
\hline 1979 & 24.46 & 24.44 & 24.14 & 23.58 & 23.90 & 23.43 & 23.76 & 22.99 & 23.45 & 23.84 & 23.79 & 24.47 & 23.85 \\
\hline 1980 & 24.32 & 24.10 & 24.48 & 24.03 & 23.65 & 23.10 & 23.41 & 23.54 & 23.58 & 22.99 & 22.51 & 22.60 & 23.53 \\
\hline 1981 & 23.43 & 22.94 & 22.73 & 22.56 & 22.18 & 22.85 & 23.07 & 23.11 & 23.36 & 22.46 & 22.39 & 23.12 & 22.85 \\
\hline 1982 & 23.60 & 22.85 & 23.57 & 23.72 & 23.96 & 22.53 & 21.40 & 21.40 & 20.75 & 21.56 & 22.08 & 21.58 & 22.42 \\
\hline 1983 & 22.24 & 22.67 & 23.31 & 23.37 & 22.40 & 22.95 & 23.43 & 23.32 & 23.31 & 23.59 & 23.77 & 23.77 & 23.18 \\
\hline 1984 & 24.20 & 24.04 & 23.73 & 23.45 & 22.74 & 23.18 & 23.05 & 23.47 & 23.83 & 23.88 & 23.72 & 23.80 & 23.59 \\
\hline 1985 & 23.96 & 24.30 & 24.33 & 24.73 & 24.77 & 25.23 & 25.12 & 25.12 & 25.31 & 25.37 & 25.46 & 25.34 & 24.92 \\
\hline 1986 & 25.51 & 25.00 & 25.29 & 25.84 & 26.09 & 26.03 & 25.99 & 26.00 & 26.06 & 26.12 & 25.78 & 26.13 & 25.82 \\
\hline 1987 & 26.43 & 26.70 & 26.62 & 26.53 & 26.33 & 25.86 & 25.69 & 25.49 & 25.32 & 25.25 & 24.86 & 24.96 & 25.84 \\
\hline 1988 & 24.15 & 24.54 & 24.70 & 24.66 & 24.69 & 24.47 & 23.92 & 23.84 & 24.16 & 23.88 & 23.85 & 23.26 & 24.18 \\
\hline 1989 & 22.96 & 23.06 & 21.75 & 21.83 & 21.00 & 21.56 & 22.20 & 21.83 & 21.17 & 20.66 & 20.49 & 20.96 & 21.62 \\
\hline 1990 & 21.59 & 21.81 & 21.31 & 20.53 & 20.34 & 20.39 & 21.49 & 21.32 & 21.86 & 22.37 & 22.77 & 22.75 & 21.54 \\
\hline 1991 & 23.24 & 23.26 & 21.03 & 21.54 & 21.54 & 18.45 & 19.18 & 20.69 & 21.65 & 21.85 & 21.96 & 21.74 & 21.34 \\
\hline 1992 & 22.36 & 22.11 & 22.86 & 23.54 & 23.33 & 24.02 & 24.29 & 24.03 & 23.83 & 24.35 & 24.19 & 24.73 & 23.64 \\
\hline 1993 & 24.19 & 24.23 & 24.03 & 24.28 & 24.40 & 24.70 & 24.58 & 24.75 & 24.84 & 24.81 & 24.79 & 24.68 & 24.52 \\
\hline 1994 & 24.80 & 24.49 & 24.49 & 24.49 & 24.62 & 24.62 & 24.80 & 24.93 & 25.04 & 25.07 & 25.22 & 24.97 & 24.80 \\
\hline 1995 & 25.15 & 25.25 & 25.09 & 25.20 & 25.20 & 25.22 & 25.09 & 25.33 & 25.29 & 25.23 & 25.28 & 25.39 & 25.23 \\
\hline 1996 & 25.42 & 25.52 & 25.65 & 25.66 & 25.50 & 25.45 & 25.56 & 25.53 & 25.52 & 25.21 & 25.25 & 25.45 & 25.48 \\
\hline 1997 & 25.44 & 25.61 & 25.52 & 25.53 & 25.59 & 25.46 & 25.53 & 25.67 & 25.58 & 25.45 & 25.30 & 25.39 & 25.51 \\
\hline 1998 & 25.40 & 25.44 & 25.52 & 24.80 & 24.31 & 24.51 & 24.80 & 24.46 & 24.78 & 25.07 & 24.85 & 24.55 & 24.87 \\
\hline 1999 & 24.14 & 24.06 & 24.23 & 24.35 & 24.26 & 24.56 & 24.73 & 24.05 & 23.52 & 23.36 & 23.26 & 23.15 & 23.97 \\
\hline 2000 & 23.41 & 23.19 & 22.94 & 23.02 & 22.33 & 21.92 & 21.42 & 22.02 & 22.25 & 22.88 & 22.04 & 22.40 & 22.49 \\
\hline 2001 & 23.04 & 23.62 & 23.99 & 22.31 & 22.94 & 23.25 & 23.40 & 22.85 & 22.83 & 22.59 & 22.82 & 23.28 & 23.08 \\
\hline 2002 & 22.40 & 23.28 & 22.81 & 22.75 & 22.86 & 22.94 & 22.54 & 21.88 & 22.40 & 22.94 & 22.45 & 22.66 & 22.66 \\
\hline 2003 & 22.82 & 23.10 & 23.27 & 23.03 & 22.51 & 22.13 & 22.52 & 22.98 & 23.24 & 22.60 & 20.88 & 22.72 & 22.65 \\
\hline 2004 & 22.73 & 23.64 & 24.02 & 24.25 & 24.33 & 24.22 & 24.14 & 24.47 & 24.78 & 25.37 & 24.37 & 24.48 & 24.23 \\
\hline 2005 & 23.47 & 24.36 & 24.49 & 24.55 & 24.04 & 24.54 & 24.38 & 24.05 & 23.31 & 24.63 & 25.00 & 25.17 & 24.33 \\
\hline 2006 & 25.13 & 25.76 & 25.78 & 25.98 & 26.05 & 26.03 & 25.77 & 25.74 & 25.66 & 26.07 & 25.85 & 25.37 & 25.77 \\
\hline 2007 & 25.98 & 25.98 & 26.14 & 26.51 & 26.46 & 26.51 & 26.47 & 26.44 & 26.52 & 26.50 & 26.45 & 26.46 & 26.37 \\
\hline 2008 & 26.33 & 26.40 & 26.42 & 26.35 & 26.32 & 26.42 & 26.48 & 26.58 & 26.60 & 26.56 & 26.68 & 26.67 & 26.48 \\
\hline 2009 & 26.69 & 26.85 & 26.85 & 26.95 & 26.95 & 26.96 & 26.98 & 26.95 & 26.97 & 27.05 & 27.01 & 27.04 & 26.94 \\
\hline 2010 & 26.89 & 26.67 & 26.56 & 26.27 & 26.32 & 26.37 & 26.40 & 26.28 & 26.21 & 26.39 & 26.10 & 26.07 & 26.38 \\
\hline 2011 & 26.14 & 26.20 & 25.89 & 25.33 & 25.73 & 24.96 & 25.35 & 25.42 & 25.52 & 25.15 & 25.57 & 25.91 & 25.60 \\
\hline 2012 & 25.84 & 25.39 & 24.77 & 25.66 & 25.64 & 25.31 & 24.67 & 24.77 & 25.28 & 25.29 & 25.31 & 25.47 & 25.28 \\
\hline 2013 & 25.69 & 25.49 & 25.04 & 25.26 & 24.37 & 24.53 & 24.82 & 25.08 & 25.12 & 25.42 & 25.21 & 24.90 & 25.08 \\
\hline 2014 & 24.82 & 24.40 & 24.58 & 24.59 & 24.89 & 24.44 & 24.67 & 25.11 & 24.55 & 24.38 & 24.21 & 23.70 & 24.53 \\
\hline 2015 & 24.01 & 24.20 & 23.80 & 24.21 & 24.46 & 24.45 & 24.89 & 24.90 & 24.81 & 24.70 & 24.79 & 24.98 & 24.52 \\
\hline 2016 & 25.28 & 25.62 & 25.52 & 25.49 & 25.58 & 25.73 & 25.63 & 25.89 & 25.88 & 26.25 & 26.32 & 26.25 & 25.79 \\
\hline 2017 & 26.36 & 26.30 & 26.51 & 26.38 & 26.20 & 26.26 & 26.11 & 25.88 & 25.66 & 26.05 & 26.47 & 26.46 & 26.22 \\
\hline 2018 & 26.48 & 26.48 & 26.42 & 26.54 & 26.55 & 26.77 & 26.80 & 26.72 & 26.68 & 26.68 & 26.57 & 26.47 & 26.60 \\
\hline
\end{tabular}

\section{Acknowledgments}

This work was partially supported by the grant RFBR № 18-02-00451. Experimentally and methodologically support the project USU "Russian national network of ground stations of cosmic rays". We are grateful to all the staff of the World Network of cosmic ray stations http://cr0.izmiran.ru/ ThankYou. 


\section{References}

Adriani, 0., Barbarino, G. C., Bazilevskaya, G. A., et al. (PAMELA Collaboration), 2011, The Astrophysical Journal, 742:102 (11pp), D0I: http://dx.doi.org/10.1088/0004-637X/742/2/102

Adriani, 0., Barbarino, G. C., Bazilevskaya, G. A., et al. (PAMELA Collaboration), 2013, The Astrophysical Journal, V.765, N.2. D0I http://dx.doi.org/10.1088/0004-637X/765/2/91

Adriani O., Barbarino, G. C., Bazilevskaya, G. A., et al. 2014, Physics Reports, 544, 4, 323.

Adriani O., Barbarino, G. C., Bazilevskaya, G. A., et al. (PAMELA Collaboration), 2017, "Ten years of PAMELA in space", Riv. Nuovo Cimento 40473 DOI: http://dx.doi.org/10.1393/ncr/i2017-10140-x

Aguilar, M., et al. (AMS Collaboration), 2015, Phys. Rev. Lett. 114, 171103, DOI: http://dx.doi.org/10.1103/PhysRevLett.114.171103

Aguilar, M., et al. (AMS Collaboration), 2018, Phys. Rev. Lett. 121, 051101, DOI: http://dx.doi.org/10.1103/physrevlett.119.251101

Alanko, K., Usoskin, I. G., Mursula, K., Kovaltsov, G. A., 2003, Adv. Space Res., 32 (4), 615-620, D0I: http://dx.doi.org/10.1016/S0273$1177(03) 00348-X$

Belov, A. V., Dorman, L. I., and Yanke, V. G., 1983, "The simplest versions of the global-spectrographical method". Proc. of 18-th ICRC, Bangalore, Vol. 10, 144-147

Belov, A. V., Gushchina, R. T., Yanke, V. G., 1998, Geomagnetism and Aeronomy, V.38, No.4, 131

CRDB (Cosmic Ray DataBase), 2020, https://tools.ssdc.asi.it/CosmicRays/chargedCosmicRays.jsp (last accessed April 8, 2021)

CR Network, 2020, nmdb: https://www.nmdb.eu/, https://www.nmdb.eu/nest/, usu: http://www.ckp-rf.ru/usu/433536, idb: http://cr0.izmiran.ru/mosc (last accessed April 8, 2021)

Di Felice, V., Pizzolotto, C., D'Urso, D., Dari, S., Navarra, D., Primavera, R., Bertucci, B., 2017,"Looking for cosmic ray data? The ASI Cosmic Ray Database" 35th ICRC, PoS 1073, Korea, https://pos.sissa.it/301/1073/pdf (last accessed April 8, 2021)

Koldobskiy, S. A., Kovaltsov, G. A., Usoskin, I. G., 2018, J. Geophys. Res. 123, 4479, DOI: https://doi.org/10.1029/2018JA025516

Koldobskiy, S. A., Bindi, V., Corti, C., Kovaltsov, G. A., Usoskin, I. G., 2019, J. Geophys. Res. 124, 2367-2379, D0I: http://dx.doi.org/ 10.1029/2018JA026340

Koldobskiy, S. A., Bindi, V., Corti, C., Kovaltsov, G. A., Usoskin, I.G., 2019, PoS ICRC 2019, id 1094

Krymskiy, G. F., Altukhov, A. M., Kuzmin, A. I., Krivoshapkin, P. A., Skripin, G. V., Chirkov, N.P., 1966, Geomagnetism and Aeronomy, V.6. №6, 991-996.

Lafferty, G. D., Wyatt, T. R., 1995, "Where to stick your data points: The treatment of measurements within wide bins" Nuclear Instruments and Methods in Physics Research A 355, 541-547. DOI: https://doi.org/10.1016/0168-9002(94)01112-5

Martucci, M., et al. 2018, Proton Fluxes Measured by the PAMELA Experiment from the Minimum to the Maximum Solar Activity for Solar Cycle 24, ApJL, 854: L2, DOI: http://dx.doi.org/10.3847/2041-8213/aaa9b2

Nagashima, K., 1971, Rep. of lonosphere and Space Res. In Japan, Vol. 25, No 3, 189

Ting, S., 2013, The Alpha Magnetic Spectrometer on the International Space Station, Nucl. Phys. B, Proc. Suppl. 243-244, 12-24

Usoskin, I. G., Alanko-Huotari, K., Kovaltsov, G. A., and Mursula, K. , 2005, J. Geophys. Res., 110, A12108, DOI: http://dx.doi.org/10.1029/2005JA011250

Usoskin, I. G., Gil, A., Kovaltsov, G. A., Mishev, A. L. and Mikhailov, V. V., 2017, J. Geophys. Res. Space Physics, 122, 3875-3887, DOI: http://dx.doi.org/10.1002/2016JA023819

Usoskin, I. G., Bazilevskaya, G. A., K., Kovaltsov, G. A., 2011, Journal of Geophysical Research: Space Physics 116, A02104, D0l: http://dx.doi.org/10.1002/2016JA023819

Yanke, V. G., Belov, A. V., Eroshenko, E. A., Trefilova, L. A., Kobelev, P. G., Gushchina, R. T., 2021, "Experimental spectrum of cosmic ray variations in the range of rigidity from 1-20 GV in the Earth's orbit according to AMS-02 data", Cosmic ray studies with neutron detectors 1 , DOI: https://dx.doi.org/10.38072/2748-3150/p4

\section{Questions and answers}

Ludwig Klein: Would you argue that the calibration of Pamela after 2012 is not reliable?

Answer: No, I wouldn't, it cannot be asserted. The PAMELA data up to and including 2009 and the AMS-02 data for the entire period are in good agreement with the data of neutron monitors, with the PAMELA data used as the base period of 2009. After a long break since 2010, there were problems with the PAMELA detector, it is evident from the increased statistical errors.

Mike Snow: The $~ 5 \%$ shift in 2010 PAMELA data is from the PAMELA collaboration or is factor that gives agreement with neutron monitor data?

Answer: A shift of $4.88 \%$ is a factor necessary for agreement with neutron monitor data, but for agreement only since 2010. The PAMELA data up to and including 2009 and the AMS-02 data for the entire period are in good agreement with the data of neutron monitors. The main conclusions of the work are not affected by the normalization of the PAMELA data after 2009 